\title{
86. Pathophysiological Studies on the Tenesmus (1st report)
}

Kazuo Nishimura, Takeo Anezaki, Keizo Sakamoto, Osamu Yasojima, Tunenori Koya, Norio Oonishi, Kiyohito TaKaO, Osamu Fujiwara AND Hiroaki KaWAKITA

The Fujita's Surgical Clinic, Kobe Medical College

Experimental tenesmus, which is thought to be the mechanism of repeated defecation, was studied in dogs. The peculiar movement of the diaphragm and the action potential of the pelvic nerve (parasympahetic nervous system) were observed during the defecation reflex. The intensity and location of stimulation that may have caused the responsable effect concerning this phenomena were definited at the intestinal canal and the peritoneum partietale of the Douglas' pouch.

The impulses of the hypogastric nerve (sympathetic nervous system) and the reflex of segmental spinal cord, furthermore, were related to tenesmus.

\section{Crossed and Uncrossed Afferent Volley at Level Medulla}

Tokihiko Tsutsumi, Kohnosuke NaKamura and Hiroshi NaKahama

Toshio Kusama and Toshiichi Terada

Department of Anatomy, Chiba University

It is well known that somatic area I \& II receive afferent volleys from not only contra-lateral but ipsi-lateral limbs. Therefore, this report was designed to secure the contra- and ipsi-lateral afferent projections at the brain stem.

Forelimb nerve stimulation evoked responses not only in ipsi-lateral but in contra-lateral Burdach and Goll nuclei. However, the shortest latency responses were only recorded from ipsi-lateral Burdach nucleus.

Similar results were obtained by stimulation of hindlimb nerve but the shorter latency responses were evoked from ipsi-lateral Goll nucleus.

These findings are compared with those of histological Nauta-Gygat method. 